\title{
Verändern Bürgerentscheide die Politik? Strukturelle und partizipatorische Auswirkungen direktdemokratischer Praxis
}

\author{
Jan Drewitz
}

\section{Fragestellung, Forschungsstand und-design}

Zu den Effekten direkter Demokratie dominiert in der Literatur die These, dass direktdemokratische Verfahren die Partizipation der Bürger aktivieren und erhöhen würden. ${ }^{1}$ Diese Annahme wird jedoch nur selten mit empirischen Zahlen belegt. Dennoch wird häufig der Schluss gezogen, dass die Ergänzung von Beteiligungsmöglichkeiten (in diesem Fall durch Instrumente direkter Demokratie) auch automatisch dazu führt, dass diese wahrgenommen werden und so die politische Partizipation erhöht wird. Diese Argumentation stützt sich empirisch fast ausschließlich auf die Untersuchungsergebnisse von Beilharz 1981. ${ }^{2}$ Als in den 1990er Jahren die neu eingeführten direktdemokratischen Verfahren in den Kommunen einer kritischen Untersuchung unterzogen wurden, sahen sich viele Autoren von Beilharz positiven Ergebnissen bestätigt, ohne jedoch ihrerseits eigene Zahlen zu erheben. Für die Schweiz liegen dagegen mehr Untersuchungen der Praxis vor, beispielsweise zu den Auswirkungen auf die Wahlbeteiligung ${ }^{3}$ oder das Vereinsengagement. ${ }^{4}$ Für Deutschland fehlen Studien zum Gegenstand der partizipatorischen und strukturellen Auswirkungen weitestgehend. Dies liegt vor allem an Problemen der Operationalisierbarkeit. So ist es schwer, politisch strukturell vergleichbare Kommunen zu finden, in denen eine repräsentative Anzahl von direktdemokratischen Verfahren vorliegt.

Die Verfahren, in deren Rahmen sich die Bürger politisch beteiligen können, sind in der Struktur der kommunalen Ebene verfasst und definiert. Dieses Angebot steht dem Wunsch und der Nachfrage nach politischer Partizipation der Bürger gegenüber. Mit der sukzessiven Einführung direktdemokratischer Verfahren auf der kommunalen Ebene bis zum Jahr 2005

1 Vgl. unter anderem Claus-Henning Obst, Chancen direkter Demokratie in der Bundesrepublik Deutschland. Zulässigkeit und politische Konsequenzen, Köln 1986, S. 320 f.; Wilfried Erbguth, Verstärkung der Elemente unmittelbarer Bürgerbeteiligung auf kommunaler Ebene, in: HansGünter Henneke (Hrsg.), Aktuelle Entwicklungen der inneren Kommunalverfassung. Zur künftigen Austarierung des Verhältnisses von effizienter Verwaltung, steuernder Vertretungskörperschaft und verstärkter Bürgerbeteiligung, Berlin 1996, S. 115 - 138, S. 134; Wilfried Marxer, „Wir sind das Volk": Direkte Demokratie - Verfahren, Verbreitung, Wirkung, Bendern 2004, S. 33; HansHerbert von Arnim, Vom Mehrwert direkter Demokratie, in: Hermann K. Heußner / Otmar Jung (Hrsg.), Mehr direkte Demokratie wagen. Volksentscheid und Bürgerentscheid: Geschichte Praxis - Vorschläge, München 2009, S. 40 - 53.

2 Vgl. Günter Beilharz, Politische Partizipation im Rahmen des $\$ 21$ der Gemeindeordnung von Baden-Württemberg, Tübingen 1981.

3 Vgl. Markus Freitag / Isabelle Stadelmann-Steffen, Wahl- oder Abstimmungsdemokratie? Der Einfluss der direkten Demokratie auf die Wahlteilnahme, in: Markus Freitag / Uwe Wagschal (Hrsg.), Direkte Demokratie. Bestandsaufnahmen und Wirkungen im internationalen Vergleich, Münster 2007, S. 189 - 216, S. 209.

4 Vgl. Markus Freitag / Aline Schniewind, Direktdemokratie und Sozialkapital: Der Einfluss der Volksrechte auf das Vereinsengagement, in: Markus Freitag / Uwe Wagschal (Hrsg.), a.a.O. (Fn. 3), S. $251-276$. 
in allen deutschen Bundesländern wurde die Angebotsseite entscheidend ergänzt - entscheidend deshalb, weil mit ihrer Einführung den Bürgern erstmals ein Instrumentarium zur Verfügung steht, mit dem sie außerhalb der turnusmäßigen Wahlen verbindlich in politische Entscheidungen eingreifen können.

Es ist zu vermuten, dass sich dadurch auch die Struktur (Angebot) und die Partizipation (Nachfrage) auf der kommunalen Ebene konkret verändern. Dies soll im Folgenden untersucht werden, und zwar auf der Angebotsseite in den Kategorien „Personal“ (Gemeinderäte/ Bürgermeister) und „Parteien“ sowie auf der Nachfrageseite hinsichtlich „Wahlbeteiligung“ und „Engagement“. Wird beispielsweise der Zugang zu Ämtern wie Stadträten und Bürgermeistern erleichtert? Verändert die Angebotserweiterung auf der Seite der Nachfrage die Höhe von Wahlbeteiligungen und politischem Engagement?

Als direktdemokratische Verfahren werden dabei alle Verfahren verstanden, die auf eine direkte Entscheidungsbeteiligung der Bevölkerung abzielen. Auf eine Unterscheidung der Einleitungsart in „von oben“ (Ratsbegehren) oder „von unten“ (Bürgerbegehren) wird verzichtet, da nur zwei der untersuchten zwölf Bürgerentscheide ausschließlich durch ein Ratsbegehren eingeleitet wurden und diesen beiden lange Diskussionsprozesse innerhalb der Gemeinden vorausgingen. Zwar wird ein Verfahren „von unten“ beispielsweise von Theo Schiller ${ }^{5}$ und Reinhard Hendler ${ }^{6}$ als wichtiges Kennzeichen von direkter Demokratie angesehen ${ }^{7}$; dennoch bescheinigt beispielsweise Norbert Kersting auch Ratsreferenden, „ähnliche deliberative Diskurse“8 zu entfalten, weshalb sie hier berücksichtigt werden sollen.

Ausgewählt wurden die beiden bayrischen Kommunen Prien am Chiemsee und Herrsching am Ammersee. Diese verfügen über eine hohe Anzahl von direktdemokratischen Verfahren auf der lokalen Ebene und eignen sich nach einer bundesweiten Recherche ${ }^{9}$ am besten für eine Untersuchung mit dem Most Similar Systems Design (MSSD) ${ }^{10}$, da die lokale politische Struktur beider Kommunen nahezu identisch ist. Die Studie bezieht alle Bürgerbegehren, Ratsbegehren und Bürgerentscheide ein, die in den beiden Kommunen ausgelöst wurden, und untersucht deren Auswirkungen auf das Angebot und die Nachfrage auf der Kommunalebene.

5 Vgl. Theo Schiller, Direkte Demokratie in Theorie und kommunaler Praxis, Frankfurt am Main 1999, S. 9.

6 Vgl. Reinhard Hendler, Vorzüge und Nachteile verstärkter Bürgerbeteiligung auf kommunaler Ebene, in: Hans-Günter Henneke (Hrsg.), a.a.O. (Fn. 1), S. 101 - 114, S. 102.

7 Hier sei ebenso auf Werner Patzelt verwiesen, für den Plebiszite, die durch Repräsentanten ausgelöst wurden, unter repräsentationstheoretischen Gesichtspunkten abzulehnen sind, da sie Entscheidungen „wegverlagern“ (S. 97) und „letztlich zum Versickern bringen“ (S. 76) würden. Werner J. Patzelt, Welche plebiszitären Instrumente könnten wir brauchen? Einige systematische Überlegungen, in: Lars P. Feld / Peter M. Huber / Otmar Jung / Christian Welzel / Fabian Wittreck (Hrsg.), Jahrbuch für direkte Demokratie 2010, Baden-Baden 2011, S. 63 - 106.

8 Norbert Kersting, Die Zukunft der lokalen Demokratie. Modernisierungs- und Reformmodelle, Frankfurt am Main 2004, S. 168.

9 Durchgeführt unter Zuhilfenahme der Datenbank der Forschungsstelle Bürgerbeteiligung und Direkte Demokratie am Institut für Politikwissenschaft der Philips-Universität Marburg, http:// www.datenbank-buergerbegehren.de (Abruf am 2. April 2012).

10 Vgl. zum Most Similar Systems Design (MSSD): Todd Landman, Issues and Methods in Comparative Politics: An Introduction, Routledge 2006, S. 70 - 78; Heidrun Abromeit / Michael Stoiber, Demokratien im Vergleich: Einführung in die vergleichende Analyse politischer Systeme, Wiesbaden 2006, S. $32 \mathrm{ff}$. 
Die Untersuchung stützt sich konzeptionell in erster Linie auf 18 qualitative Experteninterviews, die im Oktober 2010 mit Vertreterinnen und Vertretern der lokal ansässigen Parteien, Wählergemeinschaften und Initiatoren der Bürgerbegehren vor Ort durchgeführt wurden und zwischen 30 und 150 Minuten dauerten. Als Ergänzung wurden außerdem die Wahlund Abstimmungsergebnisse sowie deren Beteiligungshöhen seit 1978 einbezogen und Zeitungsartikel der lokalen Presse gesichtet. In beiden Kommunen musste von stimulierenden Partizipationswirkungen der Verfahren ausgegangen werden, da die überwiegende Anzahl der eingeleiteten Begehren zu einem Erfolg im Sinne der Initiatoren geführt hat ${ }^{11}$ : In Prien waren es drei (von drei) und in Herrsching vier (von fünf) der Bürgerbegehren, die in einen Entscheid mündeten.

Die Marktgemeinde Prien am Chiemsee befindet sich circa 60 Kilometer südöstlich von München im Landkreis Rosenberg; die Gemeinde Herrsching am Ammersee liegt circa 30 Kilometer südwestlich von München im Landkreis Starnberg. Beide gehören zum Regierungsbezirk Oberbayern im Bundesland Bayern. Prien zählt 10.292 Einwohner (wahlberechtigt 2008: 8.054), Herrsching 10.053 (7.571). Beide sind kleinstädtische Gemeinden, die über sechs verschiedene Parteien und Wählergemeinschaften im Gemeinderat verfügen. Folgende direktdemokratische Verfahren wurden in diesen Kommunen durchgeführt:

\begin{tabular}{|c|c|c|c|c|c|}
\hline Jahr & Art & ausgelöst durch & Initiatoren & Thema & Ausgang \\
\hline 1997 & $\begin{array}{l}\text { Bürger- } \\
\text { entscheid }\end{array}$ & $\begin{array}{l}\text { Bürgerbegehren } \\
\text { „Rettet den Wendel- } \\
\text { steinplatz“; Ratsbe- } \\
\text { gehren }\end{array}$ & $\begin{array}{l}\text { Überparteiliche } \\
\text { Wählergemein- } \\
\text { schaft (ÜWG); } \\
\text { Gemeinderat }\end{array}$ & $\begin{array}{l}\text { Gegen privates } \\
\text { Bauvorhaben; } \\
\text { Umgestaltung eines } \\
\text { öffentlichen Platzes }\end{array}$ & $\begin{array}{l}\text { Positiv im Sinne } \\
\text { des Bürgerbegeh- } \\
\text { rens }\end{array}$ \\
\hline 1997 & $\begin{array}{l}\text { Bürger- } \\
\text { begehren }\end{array}$ & $\begin{array}{l}\text { Bürgerbegehren } \\
\text { „Neue Verkehrs- } \\
\text { führung in Prien“ }\end{array}$ & $\begin{array}{l}\text { Private Initiati- } \\
\text { ve }\end{array}$ & Verkehrsführung & $\begin{array}{l}\text { Durch den } \\
\text { Gemeinderat für } \\
\text { ungültig erklärt }\end{array}$ \\
\hline 1997 & $\begin{array}{l}\text { Bürger- } \\
\text { begehren }\end{array}$ & $\begin{array}{l}\text { Bürgerbegehren } \\
\text { „Für Erhalt des } \\
\text { Warmbades“ } \\
\end{array}$ & Bürgerinitiative & $\begin{array}{l}\text { Gegen Bauvorha- } \\
\text { ben eines Spaß- } \\
\text { bades }\end{array}$ & $\begin{array}{l}\text { Durch den } \\
\text { Gemeinderat für } \\
\text { ungültig erklärt } \\
\end{array}$ \\
\hline 1999 & $\begin{array}{l}\text { Bürger- } \\
\text { entscheid }\end{array}$ & $\begin{array}{l}\text { Bürgerbegehren } \\
\text { „Keine Seebühne in } \\
\text { Prien“; Ratsbegehren }\end{array}$ & $\begin{array}{l}\text { Bürgerinitiative; } \\
\text { Gemeinderat }\end{array}$ & $\begin{array}{l}\text { Gegen Kultur-/ } \\
\text { Bauprojekt } \\
\text { „Seebühne“ } \\
\end{array}$ & $\begin{array}{l}\text { Positiv im Sinne } \\
\text { des Bürgerbegeh- } \\
\text { rens }\end{array}$ \\
\hline 2005 & $\begin{array}{l}\text { Bürger- } \\
\text { entscheid }\end{array}$ & $\begin{array}{l}\text { Bürgerbegehren „Er- } \\
\text { haltet unser Strand- } \\
\text { bad“; Ratsbegehren }\end{array}$ & $\begin{array}{l}\text { Bürgerinitiative; } \\
\text { Gemeinderat }\end{array}$ & $\begin{array}{l}\text { Gegen Bauvorha- } \\
\text { ben einer „See- } \\
\text { sauna“" }\end{array}$ & $\begin{array}{l}\text { Positiv im Sinne } \\
\text { des Bürgerbegeh- } \\
\text { rens }\end{array}$ \\
\hline $2011^{*}$ & $\begin{array}{l}\text { Bürger- } \\
\text { entscheid }\end{array}$ & Ratsbegehren & Gemeinderat & $\begin{array}{l}\text { Gegen Projekt und } \\
\text { Bauvorhaben } \\
\text { „Hackschnitzel- } \\
\text { werk“ }^{\text {a }}\end{array}$ & $\begin{array}{l}\text { Negativ im Sinne } \\
\text { des Ratsbegehrens }\end{array}$ \\
\hline \multicolumn{6}{|c|}{$\begin{array}{l}\text { * Dieser Bürgerentscheid wurde erst nach Abschluss der Untersuchung durchgeführt und konnte deshalb } \\
\text { nur bei der Analyse der Entwicklung der Abstimmungshöhen einbezogen werden. } \\
\text { Quelle: Eigene Zusammenstellung. }\end{array}$} \\
\hline
\end{tabular}

11 Vgl. Stefanie Lackner, Willensbildungsprozesse im Rahmen von Bürgerentscheiden, in: Theo Schiller (Hrsg.), a.a.O. (Fn. 5), S. 69 - 113, S. 98. 


\begin{tabular}{|c|c|c|c|c|c|}
\hline Jahr & Art & ausgelöst durch & Initiatoren & Thema & Ausgang \\
\hline 1997 & $\begin{array}{l}\text { Bürger- } \\
\text { entscheid }\end{array}$ & $\begin{array}{l}\text { Bürgerbegehren } \\
\text { „Seniorenstift" }\end{array}$ & Private Initiative & $\begin{array}{l}\text { Gegen privates } \\
\text { Bauvorhaben; } \\
\text { für kommunales } \\
\text { Bauprojekt }\end{array}$ & $\begin{array}{l}\text { Positiv im Sinne } \\
\text { des Bürgerbegeh- } \\
\text { rens }\end{array}$ \\
\hline 1997 & $\begin{array}{l}\text { Bürger- } \\
\text { entscheid }\end{array}$ & $\begin{array}{l}\text { Bürgerbegehren } \\
\text { „Für Trinkwasserei- } \\
\text { genversorgung“; } \\
\text { Bürgerbegehren } \\
\text { „Für Trinkwasserei- } \\
\text { genversorgung unter } \\
\text { Berücksichtigung } \\
\text { weiterer Varianten }\end{array}$ & $\begin{array}{l}\text { NGO, Bürgerin- } \\
\text { itiative, Bündnis } \\
\text { 90/Die Grünen, } \\
\text { FW, Private Ini- } \\
\text { tiative (unter- } \\
\text { stützt durch } \\
\text { CSU) }\end{array}$ & $\begin{array}{l}\text { Trinkwasserversor- } \\
\text { gung durch eige- } \\
\text { nen Brunnen } \\
\text { oder/und zusätzli- } \\
\text { cher Anschluss an } \\
\text { Wasserverbund }\end{array}$ & $\begin{array}{l}\text { Positiv im Sinne } \\
\text { des } 1 \text {. Bürgerbe- } \\
\text { gehrens }\end{array}$ \\
\hline 2001 & $\begin{array}{l}\text { Bürger- } \\
\text { entscheid }\end{array}$ & Ratsbegehren & $\begin{array}{l}\text { Gemeinderat } \\
(\mathrm{CSU})\end{array}$ & $\begin{array}{l}\text { Vergrößerung } \\
\text { Gewerbegebiet }\end{array}$ & $\begin{array}{l}\text { Negativ im Sinne } \\
\text { des Ratsbegehrens }\end{array}$ \\
\hline 2004 & $\begin{array}{l}\text { Bürger- } \\
\text { entscheid }\end{array}$ & $\begin{array}{l}\text { Bürgerbegehren } \\
\text { „Erhalt der Natur am } \\
\text { Herrschinger Moos" }\end{array}$ & $\begin{array}{l}\text { NGO, Bürger- } \\
\text { initiative, Bünd- } \\
\text { nis 90/Die Grü- } \\
\text { nen, FW }\end{array}$ & $\begin{array}{l}\text { Für Erhalt Natur- } \\
\text { gebiet; gegen } \\
\text { Ausweitung des } \\
\text { Gewerbegebiets }\end{array}$ & $\begin{array}{l}\text { Negativ im Sinne } \\
\text { des Bürgerbegeh- } \\
\text { rens }\end{array}$ \\
\hline 2006 & $\begin{array}{l}\text { Bürger- } \\
\text { entscheid }\end{array}$ & $\begin{array}{l}\text { Bürgerbegehren "Ge- } \\
\text { gen luxuriöse Skater- } \\
\text { anlage“ }\end{array}$ & $\begin{array}{l}\text { Private Initiative } \\
\text { (unterstützt } \\
\text { durch CSU) }\end{array}$ & $\begin{array}{l}\text { Gegen Kultur-I } \\
\text { Bauprojekt "Ska- } \\
\text { teranlage“ am See }\end{array}$ & $\begin{array}{l}\text { Positiv im Sinne } \\
\text { des Bürgerbegeh- } \\
\text { rens }\end{array}$ \\
\hline 2008 & $\begin{array}{l}\text { Bürger- } \\
\text { entscheid }\end{array}$ & $\begin{array}{l}\text { Bürgerbegehren } \\
\text { "Gegen Parkhaus } \\
\text { am Bahnhof" }\end{array}$ & $\begin{array}{l}\text { Private Initiative } \\
\text { (unterstützt } \\
\text { durch CSU, } \\
\text { FBU) }\end{array}$ & $\begin{array}{l}\text { Gegen Bauvor- } \\
\text { haben eines Park- } \\
\text { hauses }\end{array}$ & $\begin{array}{l}\text { Positiv im Sinne } \\
\text { des Bürgerbegeh- } \\
\text { rens }\end{array}$ \\
\hline
\end{tabular}

\section{Auswirkungen auf die kommunalen Strukturen}

\subsection{Parteien und Repräsentanten}

Obwohl alle politischen Lager und auch die Gruppen außerhalb des Gemeinderats an den vielfältigen Bürgerbegehren und -entscheiden beteiligt waren, ist in Prien und Herrsching weder bei den Parteien und Wählergemeinschaften noch in den zivilgesellschaftlichen Gruppen zu erkennen, dass daraus zählbare Mitgliederzuwächse resultierten.

In Prien berichteten die CSU und die Überparteiliche Wählergemeinschaft (ÜWG) vereinzelt von neuen Mitgliedern, die eventuell auf die Begehren zurückzuführen seien, dies wurde jedoch in den Interviews so wenig konkret geäußert, dass hiervon nicht auszugehen ist. Weder die ÜWG noch die Bürger für Prien (BfP), die mit den jeweiligen Bürgerbegehren eng verbunden waren, konnten Erfolge verzeichnen, die über Sympathie oder Wertschätzung für ihr Engagement hinausgingen. Auch die Mitglieder von Mehr Demokratie für Prien $(\mathrm{MDfP})^{12}$, die bei der Benennung ihrer Wählergemeinschaft auf den programmatischen

12 Diese Wählergemeinschaft hat sich 2011 aufgelöst und unter dem Namen „Miteinander für Prien“ neu gegründet. 
Namen des Vereins „Mehr Demokratie“ zurückgriffen und sich bei den Begehren engagierten, hatten sich nach den Erfolgen bei den Begehren und der Kommunalwahl 2008 einen Mitgliederzuwachs erhofft. Diese Hoffnung erfüllte sich nicht.

Auch in Herrsching gilt der Befund, dass es keine Personen aus dem Initiatorenkreis der Bürgerbegehren gab, die ihr Engagement als „Sprungbrett“ für den Einstieg in die kommunalpolitische Arbeit nutzten. Entweder waren diese Bürger schon vorher politisch aktiv oder ihr Engagement beschränkte sich ausschließlich auf den Zeitraum der Begehren. Nur in einem Fall gab es eine Person, die sich in der Herrschinger Initiative Trinkwasser (HIT) beim Begehren 1997 stark engagiert hatte, im Anschluss daran einer Wählergemeinschaft beitrat und längere Jahre für diese im Gemeinderat saß.

In keiner der beiden Gemeinden führte somit die Ausweitung des Partizipationsangebots zu einer Erhöhung der Nachfrage seitens der Bürger. Dieser Befund steht damit im Widerspruch zu dem Ergebnis von Günter Beilharz, der „in 72 Prozent der Fälle eine qualitative und quantitative Veränderung der kommunalpolitischen Aktivitäten bei den Initiatoren "13 festgestellt hatte. Diese Diskrepanz muss darauf zurückgeführt werden, dass in Prien und Herrsching die meisten der Initiatoren schon vor dem Bürgerbegehren einer Partei oder Wählergemeinschaft angehörten.

Differenzierter müssen jedoch die Veränderungen bezüglich der Zusammensetzung beziehungsweise der Machtverschiebung der bestehenden Akteure im Gemeinderat betrachtet werden. Ein Vergleich der Wahlergebnisse vor (1996) und nach (2008) der Anwendung direktdemokratischer Verfahren zeigt in Prien geringfügige Veränderungen (siehe Abbildung 1).

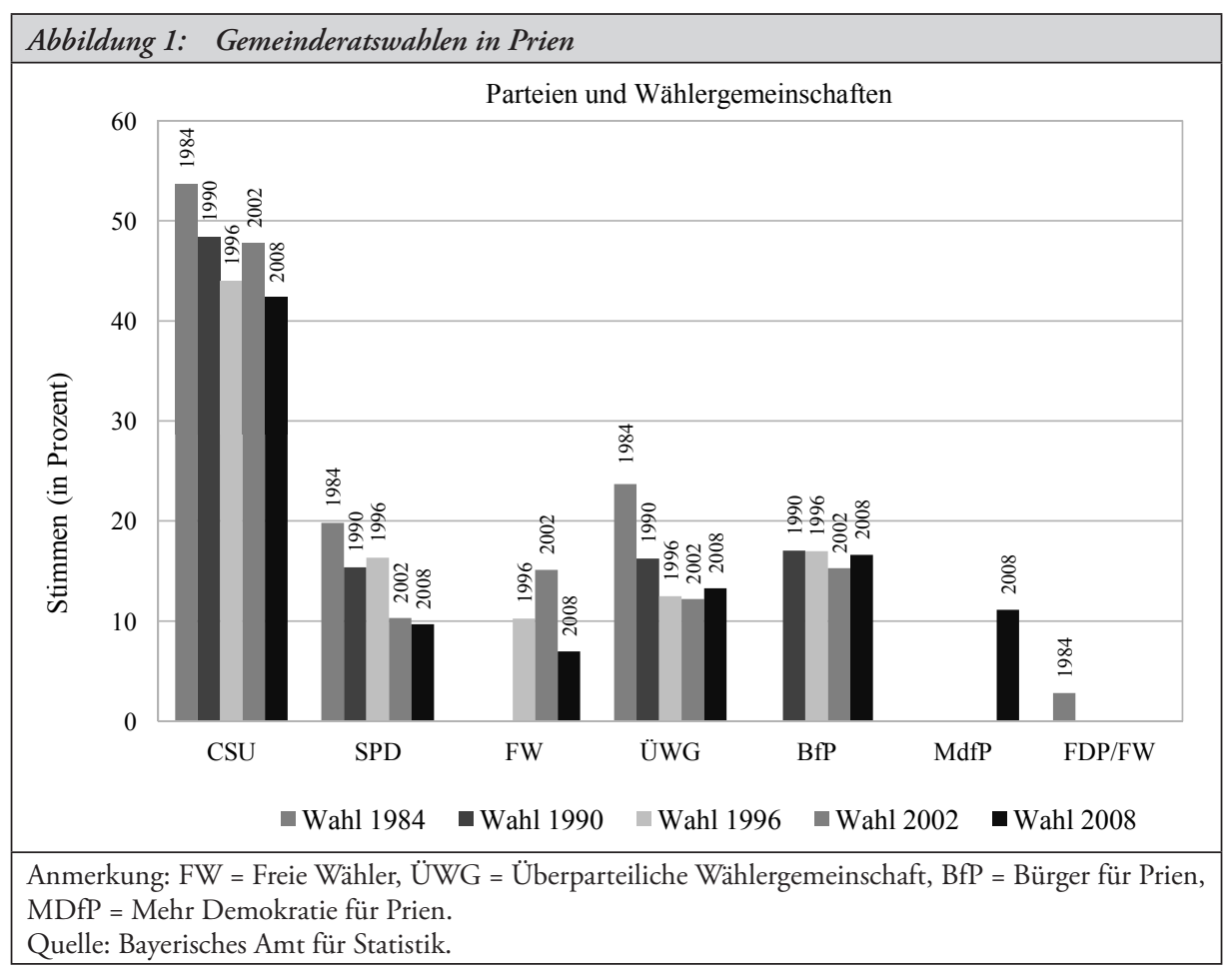

13 Günter Beilharz, a.a.O. (Fn. 2), S. 135. 


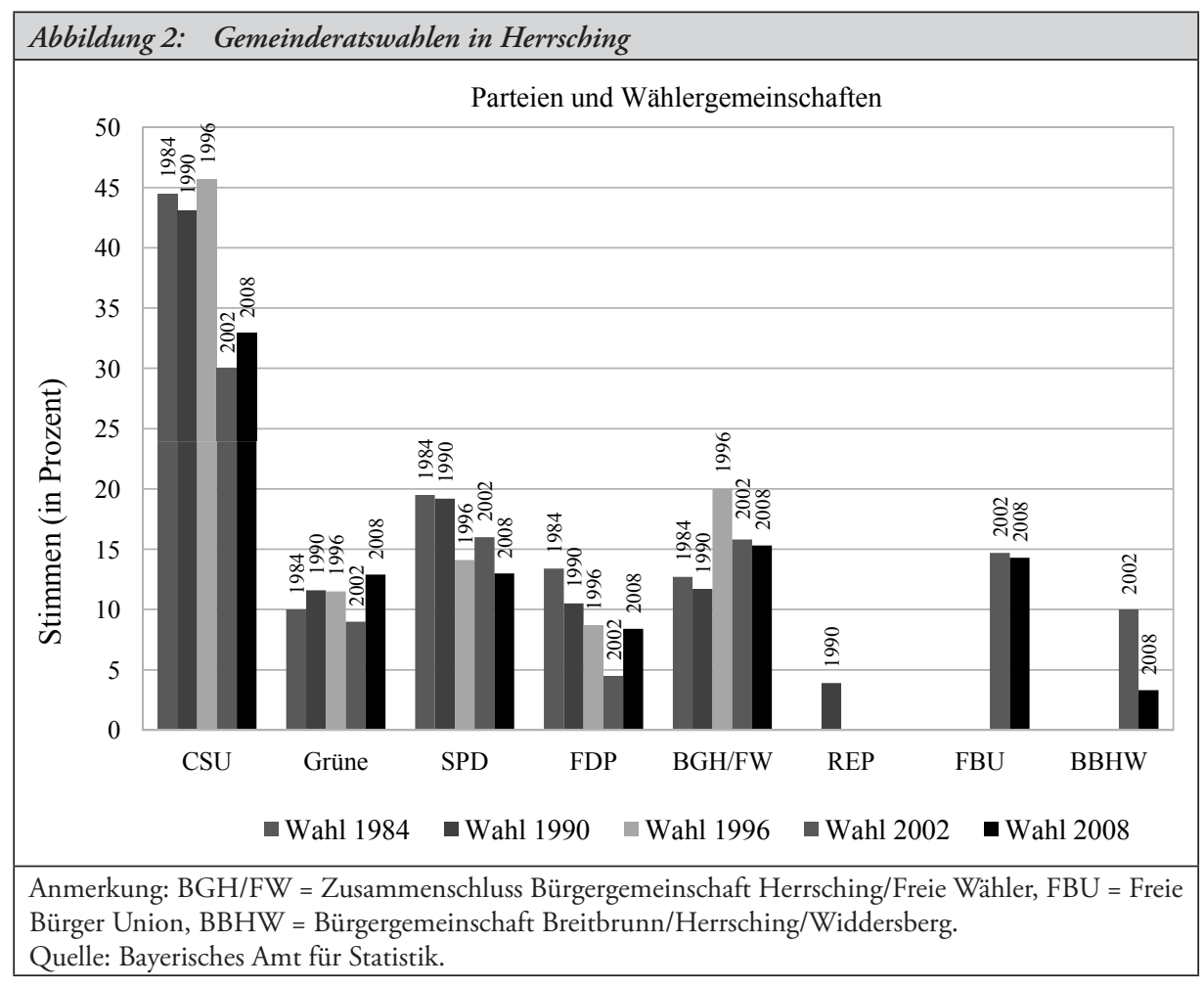

Innerhalb dieser zwölf Jahre verlor die SPD bei Kommunalwahlen sechs Prozent, die Freien Wähler (FW) drei und die CSU zwei Prozentpunkte; diese Stimmen erhielt die 2006 neu gegründete Wählergemeinschaft MDfP. Die BfP, deren Mitglieder sich stark bei den Begehren 1999 und 2005 engagiert hatten, konnte insgesamt keine Zuwächse verzeichnen und verlor 2002 sogar einige Stimmen. Auch die ÜWG, die das Begehren 1997 initiiert hatte, erhielt in der anschließenden Kommunalwahl nicht mehr Stimmen. Einzig die MDfP, deren Gründungsmitglieder ebenfalls aus der Gruppe der Initiatoren der Begehren von 1999 und 2005 kamen, scheint stark von den Bürgerentscheiden profitiert zu haben. Möglicherweise wurde dies aber auch von dem parteilosen Bürgermeisterkandidaten S. ${ }^{14}$ begünstigt, den viele Einwohner als Kandidaten von MDfP wahrnahmen.

Die Herrschinger Bürgerbegehren sind bis auf das erste 1997 unter maßgeblichem Einfluss der Parteien und Wählergemeinschaften initiiert worden. Auf diesem Wege konnten diese in der Gemeinde Sachentscheidungen unter Umgehung des Gemeinderats herbeiführen, wenn in diesem Gremium keine eigene Mehrheit bestand. Bei den ersten Begehren nutzten dies Bündnis 90/Die Grünen und der Zusammenschluss Bürgergemeinschaft Herrsching/Freie Wähler (BGH/FW) in Zusammenarbeit mit zivilgesellschaftlichen Gruppen, später dann die CSU und die Freie Bürger Union (FBU), indem sie Privatpersonen maßgeblich unterstützten.

14 Alle Personennamen werden, da sie für die Untersuchung nicht relevant sind, aus persönlichkeitsrechtlichen Gründen abgekürzt. 
Trotz der starken Inanspruchnahme dieser Instrumente durch die Parteien und Wählergemeinschaften sind bei den Involvierten nur geringfügige Veränderungen bei den nachfolgenden Wahlergebnissen zu beobachten (siehe Abbildung 2). So konnten Bündnis 90/Die Grünen und die BGH/FW keine Stimmzuwächse nach dem erfolgreichen Bürgerbegehren 1997 und der ebenfalls erfolgreichen Mobilisierung gegen den Bürgerentscheid 2001 verzeichnen; vielmehr verloren sie 2002 bei der Kommunalwahl Stimmenanteile im Vergleich zur vorangegangenen Wahl (Bündnis 90/Die Grünen: -1,5 Punkte; BGH/FW: -4,2 Punkte). Die CSU, die 1997 das zweite Begehren zur Wassereigenversorgung initiiert hatte, verlor bei der anschließenden Wahl 15,7 Prozentpunkte. Es kann aber vermutet werden, dass diese Stimmen fast geschlossen der von einem Ex-CSU-Ratsmitglied neu gegründeten FBU zukamen, die ad hoc 14,7 Prozent erreichte. Der Austritt dieses Ratsmitgliedes erfolgte unter anderem wegen des negativen Ausgangs des Ratsbegehrens 2001, so dass dieses Ergebnis auch mit dem direktdemokratischen Verfahren verknüpft werden muss. Durch das erfolgreiche Bürgerbegehren der CSU zur Skateranlage 2005 konnten ebenfalls keine nennenswerten Stimmzuwächse bei der Kommunalwahl 2008 erzielt werden; die CSU verzeichnete lediglich einen Anstieg von 2,9 Prozentpunkten gegenüber der Wahl zuvor.

Somit konnte in Prien und Herrsching nur in sehr geringem Maße ein Einfluss der direktdemokratischen Verfahren auf die Kommunalwahlergebnisse der an den Begehren beteiligten Parteien und Wählergemeinschaften festgestellt werden (in Prien für MDfP positiv; in Herrsching für die CSU negativ). Die Annahme von Andreas Paust, dass Parteien und Wählergemeinschaften Profit aus einer aktiven Unterstützung von Begehren schlagen können ${ }^{15}$, konnte deshalb nur teilweise bestätigt werden.

Direktdemokratischen Verfahren wird unterstellt, dass sie dazu beitragen, die Responsivität zwischen Ratsmitgliedern und Einwohnern zu erhöhen ${ }^{16}$, da durch das Damoklesschwert der Bürgerentscheide, das über den Ratsmitgliedern hinge, diese dazu gezwungen wären, vor weitreichenden Entscheidungen Kontakt mit den Bürgern aufzunehmen und diese umfassender in den Entscheidungsprozess einzubeziehen. ${ }^{17}$

In Prien wiederholen sich, trotz der zeitlichen Nähe und denselben Gemeinderatsmitgliedern, im Vorfeld der Gemeindeprojekte die Intransparenz und die fehlende Einbindung der Einwohner. Zwar versprach Bürgermeister K. nach dem Bürgerentscheid 1999 gegen eine Seebühne: „Vor wichtigen Entscheidungen werden wir die Bevölkerung in Zukunft besser informieren und am Diskussionsprozess beteiligen "18, doch ließ er in der regionalen Presse ebenfalls verlauten: „Immerhin hat der Entscheid nach der neusten Rechtslage nur ein Jahr aufschiebende Wirkung. "19 Dieses zweite Zitat belegt, dass ihm das Bürgervotum letztendlich unwichtig war - ein Damoklesschwert scheint er jedenfalls nicht über seinem Haupt empfunden zu haben. Es ist daher nicht verwunderlich, dass das Projekt - nach diesem öf-

15 Vgl. Andreas Paust, Wirkungen der direkten Demokratie auf das kommunale Parteiensystem, in: Theo Schiller / Volker Mittendorf(Hrsg.), Direkte Demokratie. Forschung und Perspektiven, Wiesbaden 2002, S. 218 - 230, S. 225.

16 Vgl. beispielsweise Stefanie Lackner, a.a.O. (Fn. 10), S. 89.

17 Vgl. Rudolf Wassermann, Vom Zuschauer zu Aktivbürger, in: Eugen Antalovsky (Hrsg.), Die Bürger und ihre Stadt: Direkte Demokratie in der Kommunalpolitik, Wien 1991, S. 87 - 101, S. 97.

18 Ludwig Fisch, Die Beatles gehen am Chiemsee baden, in: SZ vom 7. Dezember 1999, S. L6 (München).

19 Christian Huber, Klares Votum gegen Seebühne, in: Chiemgau-Zeitung vom 6. Dezember 1999, keine Seite, da die Kopie in den Unterlagen der Gemeindeverwaltung nicht paginiert wurde. 
fentlichen Fußtritt des eindeutigen Bürgervotums - gesetzeswidrig durch die Gemeindeverwaltung auf dem Gelände einer Nachbargemeinde weiterverfolgt wurde. Erst die unter Bürgermeister F. zaghaft begonnenen Bürgerworkshops und -runden, die unter dem aktuellen Bürgermeister S. weiter ausgebaut wurden, lassen darauf schließen, dass nicht unbedingt direktdemokratische Instrumente für die zusätzlichen Partizipationsangebote innerhalb der politischen Struktur verantwortlich waren, sondern dass solche Angebote vielmehr von den jeweiligen Entscheidungsträgern abhängen. Hierbei ist jedoch zu beachten, dass die Bürgermeister, unter denen erste Bürgerworkshops in Prien eingeführt wurden, von den Wählergemeinschaften unterstützt wurden, die sich auch für die Bürgerbegehren eingesetzt hatten. Um deren Unterstützung zu finden, waren die Bürgermeister also quasi dazu gezwungen, neue Partizipationsangebote bereitzustellen, um den vorher in den Bürgerbegehren zum Ausdruck gebrachten Bedarf auch zu bedienen.

Für Herrsching könnte durch das Auslösen des Ratsbegehrens 2001 und wegen einiger in diesem Zuge gemachter Äußerungen von Gemeinderäten der CSU vermutet werden, dass der Gemeinderat mit dem ausgelösten Bürgerentscheid den Einwohnern eine wichtige kommunale Entscheidung überlassen wollte. Diese Vermutung kann aber mit hoher Wahrscheinlichkeit widerlegt werden, da nur zwei Jahre später über dasselbe Thema erneut ein Gemeinderatsbeschluss gefasst wurde, obwohl die Bürger eigentlich 1997 beim Entscheid zur Wassereigenversorgung und 2001 zum Gewerbegebiet eindeutig eine gegenteilige Position zum Ausdruck gebracht hatten. Auch die Aussage eines Gemeinderates der CSU im Jahr 2003, man sei schließlich dafür gewählt worden, „um im Namen der Bürger Entscheidungen zu treffen" ${ }^{20}$, bestätigt die Vermutung, dass es bei diesem Ratsbegehren nicht darum ging, den Bürgern eine wichtige kommunalpolitische Entscheidung zu überlassen.

In beiden Kommunen zeigt die hohe Anzahl von Bürgerbegehren zur Verhinderung kommunaler Bauprojekte, dass es auch im Anschluss an die Bürgerentscheide den Gemeindeverwaltungen und Repräsentanten nicht gelungen ist, die Bürger stärker in Bauprojekte einzubeziehen. Exemplarisch ist dafür der Herrschinger Bürgerentscheid 2006, bei dem es einigen Initiatoren nebenbei auch darum ging, die Bürgermeisterin abzustrafen. Interessant ist dabei, dass drei der Begehren in die Zeit der Bürgermeisterin H. (BGH/FW) fielen, die zuvor selbst aktiv Bürgerbegehren initiiert hatte. Dies zeigt, dass nicht unbedingt alle Gründe für die Initiierung von Bürgerbegehren verschwunden sein müssen, wenn ehemalige Initiatoren später selbst die entscheidenden Positionen besetzen.

Im Zusammenhang mit diesen ausgebliebenen Responsivitätswirkungen fiel zudem das eklatante Unwissen über die rechtlich verbindlichen Konsequenzen direktdemokratischer Instrumente bei Herrschinger Gemeinderatsmitgliedern auf. Die Süddeutsche Zeitung befand: „Die Debatte gestaltete sich deshalb so kontrovers, da auch die CSU-Fraktion, aus deren Mitte der Antrag für das Ratsbegehren stammte, nicht recht wusste, welche kommunalpolitischen Folgen ein Begehren nach sich ziehen wird." ${ }^{21}$ Und noch eine Woche vor einem Bürgerentscheid war ein Gemeinderatsmitglied der SPD der Fehlmeinung, „das Bür-

20 Gert Sarring, Ratsbegehren findet keine Mehrheit, in: SZ vom 15. Oktober 2003, S. R5 (Starnberg).

21 Wolfgang Prochaska, Votum für Ratsbegehren, in: SZ vom 2. August 2000, S. R5 (Starnberg). 
gerbegehren (sic! ${ }^{22}$ ) ist formal nur eine Befragung. Der Gemeinderat muss entscheiden“23. Diese Äußerung ist umso erstaunlicher, als zu diesem Zeitpunkt bereits zwei Bürgerentscheide in Herrsching stattgefunden hatten und deren rechtliche Bindungswirkung hätte bekannt sein müssen. Auch noch im Jahre 2008, nach mittlerweile vier erfolgreichen Bürgerbegehren und einem Ratsbegehren, musste die CSU-Fraktion einen Antrag für ein Ratsbegehren in einer Gemeinderatssitzung zurückziehen, weil sie schlicht nicht wusste, dass es dafür einer ausformulierten Frage bedurfte. ${ }^{24}$

Es mag sein, dass dies vor allem der Situation der ehrenamtlichen Gemeinderatsmitglieder in kommunalen Räten geschuldet ist, die diese Gremienarbeit neben ihrem Beruf und meist weiteren ehrenamtlichen Tätigkeiten ausüben. Dennoch enthüllt gerade die Unkenntnis über Instrumente, die die Bürger direkt einbeziehen würden, deren Unwichtigkeit für die Fraktionen oder die jeweiligen Ratsmitglieder.

\subsection{Bürgermeisterwahlen}

Auffällig ist die ähnliche Entwicklung in der Ämtervergabe, die beide Gemeinden in Bezug auf ihre Bürgermeister anscheinend synchron vollzogen haben. 2002 wurde in beiden Kommunen ein CSU-Bürgermeister von einem Kandidaten der Freien Wähler (FW) abgelöst. ${ }^{25}$ 2008 wurden diese beiden Bürgermeister der FW dann von einem parteilosen Kandidaten ersetzt. ${ }^{26}$ In beiden Fällen beschreibt dies eine Entwicklung ausgehend von einem Angehörigen einer in Bayern dominierenden Partei, weiter zu einem Angehörigen einer explizit parteifreien Wählergemeinschaft, hin zu einer Person, die gar nicht mehr in einer Gruppe organisiert ist. Es ist deutlich zu erkennen, dass die vorangegangenen direktdemokratischen Verfahren nicht nur, wie schon angedeutet, im Vorfeld Einfluss auf die Auswahl der Bürgermeisterkandidaten gehabt haben, sondern dass diese auch die Wahl oder Abwahl von Personen begünstigt haben.

In Prien stellte sich der seit 24 Jahren im Amt befindliche Bürgermeister K. 2002 erneut zur Wahl. Trotz dieser langen Zeit als oberster Repräsentant der Gemeinde verlor der CSUPolitiker bei der Kommunalwahl gegen den Kandidaten der FW. Nachdem zwei Bürgerentscheide gegen K.'s ausgegangen waren und die Gemeindeverwaltung unter seiner Führung offen gegen den eindeutigen zweiten Bürgerentscheid die Seebühne im Nachbarort zu realisieren versuchte, ist es sehr wahrscheinlich, dass er vor diesem Hintergrund abgewählt wurde. Einschätzungen von Interviewten bestätigen diese Annahme. Obwohl K. 24 Jahre im Amt war, führten erst die Bürgerentscheide zu einem Amtsverlust.

In die Amtszeit seines Nachfolgers F. fiel 2005 ebenfalls per Bürgerentscheid ein deutliches Votum gegen die Politik des Gemeinderats und des Bürgermeisters. Dass F., genauso wie zuvor K., nur aufgrund des Entscheids 2008 wieder abgewählt worden ist, darf trotzdem

22 Korrekt hätte es hier „der Bürgerentscheid“ heißen müssen. Es ist allerdings auch nicht auszuschließen, dass dieser Fehler dem Redakteur Gert Sarrig unterlief.

23 Gert Sarrig, Rede und Gegenrede, in: SZ vom 15. Januar 2001, S. R5 (Starnberg).

24 CSU nimmt zweiten Anlauf, in: SZ vom 6. Februar 2008, S. R4 (Starnberg).

25 In Prien gab es eine Abwahl, in Herrsching trat der langjährige CSU-Bürgermeister nicht mehr zur Wahl an, der Neukandidat der CSU wurde nicht gewählt.

26 In Prien durch einen „Ortsfremden“, in Herrsching durch ein ehemaliges Gemeinderatsmitglied von der CSU. 
nicht vermutet werden, auch wenn der Entscheid dazu beigetragen haben könnte. Viel wichtiger für diese Abwahl war die Tatsache, dass F. kurz nach dem Bürgerentscheid von den FW zur CSU übertrat, da er nicht mehr den Eindruck hatte, „etwas bewegen zu können“ 27 . Dies brachte vor allem die Wähler der Wählergemeinschaften gegen ihn auf, die ihn zuvor als Kandidaten gegen die CSU unterstützt hatten. Ebenfalls trug zu seiner Abwahl bei, dass er die zuvor angekündigte Transparenz nicht einlösen konnte und seine „schwache Amtsführung "28 auf Ablehnung stieß.

2006 gründete sich die Wählergemeinschaft MDfP, deren Mitglieder größtenteils in die vorherigen beiden Bürgerbegehren involviert waren. Die Gründung wurde überwiegend aus wahltaktischen Motiven verfolgt, und die Mitglieder waren fast alle schon vorher in anderen Wählergemeinschaften aktiv. Auf Initiative der MDfP suchten FW, BfP und SPD vor der Kommunalwahl per Annonce gemeinsam nach einem auswärtigen Kandidaten für das Amt des Bürgermeisters, da der vorher unterstützte Kandidat F. (siehe oben) für sie nicht mehr in Frage kam. Die gesuchte Person sollte explizit parteilos sein, vor allem einen offenen, transparenten Umgang pflegen und über wirtschaftliches und juristisches Wissen verfügen. In der Kommunalwahl 2008 setzte sich im zweiten Wahlgang eben dieser Kandidat S. gegen den Amtsinhaber F. durch. Dies war vor allem deshalb überraschend, weil mit dem auswärtigen S. ein „Zuagroaster“ (Zugereister) den Wahlkampf in Prien gewann. ${ }^{29}$ Der Status der Parteilosigkeit war im Fall S. jedoch wahrscheinlich nicht entscheidend, da ihn die Priener im Wahlkampf eher als Mitglied von MDfP wahrnahmen. Die Bürgerbegehren in Prien haben damit in einem Fall eindeutig für einen Wechsel im Bürgermeisteramt gesorgt und darüber hinaus auf das Auswahlangebot der Kandidaten Einfluss genommen.

Auch in Herrsching können die Bürgerbegehren als ein Faktor bei der Veränderung der Besetzung des Bürgermeisteramts genannt werden. 2002 trat der langjährige CSU-Bürgermeister W. aus Altersgründen nicht mehr an, so dass die CSU einen neuen Kandidaten benötigte. Im Zuge des von der CSU initiierten Ratsbegehrens 2001 hatte es innerhalb der Ortsgruppe Streit gegeben, so dass der zweite Bürgermeister (Hauptinitiator des Ratsbegehrens im Gemeinderat) aus der CSU austrat und eine eigene Wählergemeinschaft (FBU) gründete. Als Folge trat bei der Bürgermeisterwahl 2002 ein CSU-Kandidat gegen einen Ex-CSU-Kandidaten (nun FBU) an. Ergebnis war, dass in der Stichwahl mit H. (BGH/FW) nicht nur eine Frau, sondern zugleich eine Person gewählt wurde, die sich zuvor bei einem Bürgerbegehren in der Gemeinde hervorgetan hatte. Auch wenn für die Untersuchung zu berücksichtigen ist, dass sich das Lager der CSU gegenseitig die Stimmen stahl, so kann doch vermutet werden, dass das Engagement von H. sowohl beim Bürgerbegehren „Wassereigenversorgung" 1997 als auch gegen das Ratsbegehren 2001 dazu beigetragen hat, ihr Profil im Ort zu schärfen, sie bekannter zu machen und ihr somit bessere Chancen auf den Bürgermeisterposten zu eröffnen: Das Bürgerbegehren war ,auch ausschlaggebend für die Bürgermeisterwahl“, so die Grünen. ${ }^{30}$

27 Heiner Effern, Viel Theater um die Seebühne, in: SZ vom 27. Dezember 2006, S. 42 (München).

28 Ebenda.

29 Die Akzeptanz eines solchen Kandidaten ist in kleinen bayerischen Gemeinden noch immer nicht selbstverständlich.

30 Freude bei den Grünen, in: SZ vom 13. April 2004, S. R5 (Starnberg); dies bestätigten ebenfalls Interviews. 
Dennoch zeigte sich in der Folgezeit, dass Personen, die auf kommunaler Ebene von einem direktdemokratischen Verfahren profitieren konnten, ebenso über diese „stolpern“ können. Während der Amtszeit H.'s fanden erneut zwei Bürgerentscheide statt, ein dritter wurde unmittelbar im Anschluss an ihre Amtsperiode eingeleitet und muss deshalb ebenfalls hinzugerechnet werden. Das erste dieser Begehren wurde 2004 in ihrem Sinne positiv beschieden, die zwei darauf folgenden negativ. Zum Begehren 2006 wurde von einigen Initiatoren explizit geäußert, dass dieses unmittelbar als Reaktion auf die Politik beziehungsweise Amtsführung von $H$. zu verstehen sei. ${ }^{31}$ Dass $H$. bei der Bürgermeisterwahl 2008 nicht einmal mehr in die Stichwahl kam, muss deshalb mehr auf ihre Arbeit als Bürgermeisterin insgesamt zurückgeführt werden als auf die für sie negativ verlaufenen Bürgerentscheide. Diese können eher als ein zusätzlicher Ausdruck der Unzufriedenheit einiger Einwohner mit ihrer Amtsführung angesehen werden. Der ihr nachfolgende Bürgermeister S. ist zwar parteilos, war aber in der Gemeinde durch seine langjährige Mitgliedschaft in der CSU bekannt. Zu seiner Wahl verhalf ihm insbesondere, dass er, anders als viele Bewerber in den Jahren zuvor, einen aktiven Haustürwahlkampf führte. Obwohl S. lange Zeit in der CSU aktiv gewesen war, ist mit ihm doch, wie in Prien, eine parteilose Person in das Bürgermeisteramt gewählt worden. In beiden Gemeinden ist diese Verschiebung zu Bürgermeistern, die immer weniger in Parteistrukturen verankert und organisiert sind, auffällig. Es wäre interessant, dieses Phänomen in anderen Gemeinden mit einer überdurchschnittlichen Anzahl direktdemokratischer Verfahren zu überprüfen.

Im Ergebnis wurden bei vier verschiedenen Bürgermeisterwahlen mindestens ein Bürgermeister aufgrund seines Umgangs mit den Bürgerentscheiden abgewählt (Prien 2002: K.), bei zwei weiteren haben sie zumindest zu einer Abwahl beigetragen (Prien 2008: F.; Herrsching 2008: H.). Bei zwei dieser Wahlen nahmen sie darüber hinaus Einfluss auf die Kandidatenauswahl (Prien 2008: S.; Herrsching 2002: Austritt des 2. Bürgermeisters aus der CSU und damit verbundene Gegenkandidatur mit einer Wählergemeinschaft). In einem weiteren Fall wurde eine Einwohnerin, die sich zuvor stark bei zwei Bürgerentscheiden engagiert hatte, in das höchste Amt der Gemeinde gewählt.

Diese Beispiele verdeutlichen, dass Bürgerbegehren und -entscheide auf die nachfolgende Wahl zum Amt des Bürgermeisters und selbst auf die Kandidatenauswahl Einfluss ausüben und auf diese Weise bestehende Strukturen der Akteure verändern können. Dies kann damit erklärt werden, dass in kleinen Gemeinden auf Kommunalebene der Bürgermeister die einzige hauptamtlich gewählte Person ist und die Ausrichtung und Umsetzung der Gemeindepolitik besonders mit ihm verbunden wird. Da Bürgerbegehren und -entscheide sich vor allem gegen die Politik der Gemeinden richten, ist zu vermuten, dass sich dies bei den anschließenden Wahlen besonders auf das Ergebnis des amtierenden Bürgermeisters auswirken wird. Die Anreicherung der bestehenden Strukturen mit direktdemokratischen Verfahren verändert somit auch die Mechanismen des Kandidatenangebots von Repräsentantenämtern, indem sie einerseits Diskussionsprozesse über die schon vorhandenen Akteure anstoßen und andererseits den Nicht-Etablierten die Möglichkeit geben können, durch den zusätzlichen Diskussionsprozess ihr Profil in der Bevölkerung zu schärfen.

31 Vgl. Patrizia Steipe, Brisante Informationen, in: SZ vom 10. November 2006, S. R4 (Starnberg). 


\section{Auswirkungen auf die Partizipation}

\subsection{Beteiligungshöhen bei Bürgerentscheiden}

Der Verlauf der Beteiligungsraten bei den durchgeführten Bürgerentscheiden in Prien und Herrsching zeigt, dass in diesen Instrumenten zwar das Potential steckt, durch mehrmalige Anwendungen höhere Beteiligungen zu erzielen, dies aber nicht automatisch eintreten muss.

In Prien lag die Beteiligung beim ersten Bürgerentscheid 1997 bei 33 Prozent und erreichte bei den nachfolgenden drei Bürgerentscheiden Werte zwischen 45 und 48,5 Prozent. Es kann vermutet werden, dass im ersten Bürgerentscheid das Instrument noch nicht ausreichend in der Bevölkerung bekannt war und sich die Mobilisierung in den darauf folgenden Entscheiden auf einer „,normalen“ Höhe eingependelt hat. Erstaunlich ist vor allem, dass der Bürgerentscheid 2011, der aus einem Ratsbegehren resultierte und somit nicht „von unten“ initiiert worden war, mit 48,5 Prozent die bislang höchste Beteiligung aufweist. In Herrsching bewegte sich die Beteiligung bei den ersten drei Bürgerentscheiden zwischen 33 und 35 Prozent. Beim Bürgerentscheid 2004 lag sie aufgrund der gleichzeitig stattfindenden Europawahl bei 54 Prozent.

Danach fiel die Beteiligung nicht wieder auf die ersten Werte zurück, sondern „nur“ auf 46 und anschließend 42 Prozent, und dies, obwohl diese Bürgerentscheide kein größeres Aufsehen erregt haben dürften als die ersten drei Entscheide. Die einzig mögliche Erklärung besteht darin, dass bei dem, der mit der Europawahl zusammenfallenden Bürgerentscheid ein Mitnahmeeffekt eintrat: Personen, die sich zuvor nicht an den Entscheiden beteiligt hatten, aber zur Europawahl gingen, stimmten bei dieser Gelegenheit erstmals auch bei einem Bürgerentscheid $\mathrm{ab}$ und wurden so motiviert, auch bei den folgenden zwei Bürgerent-

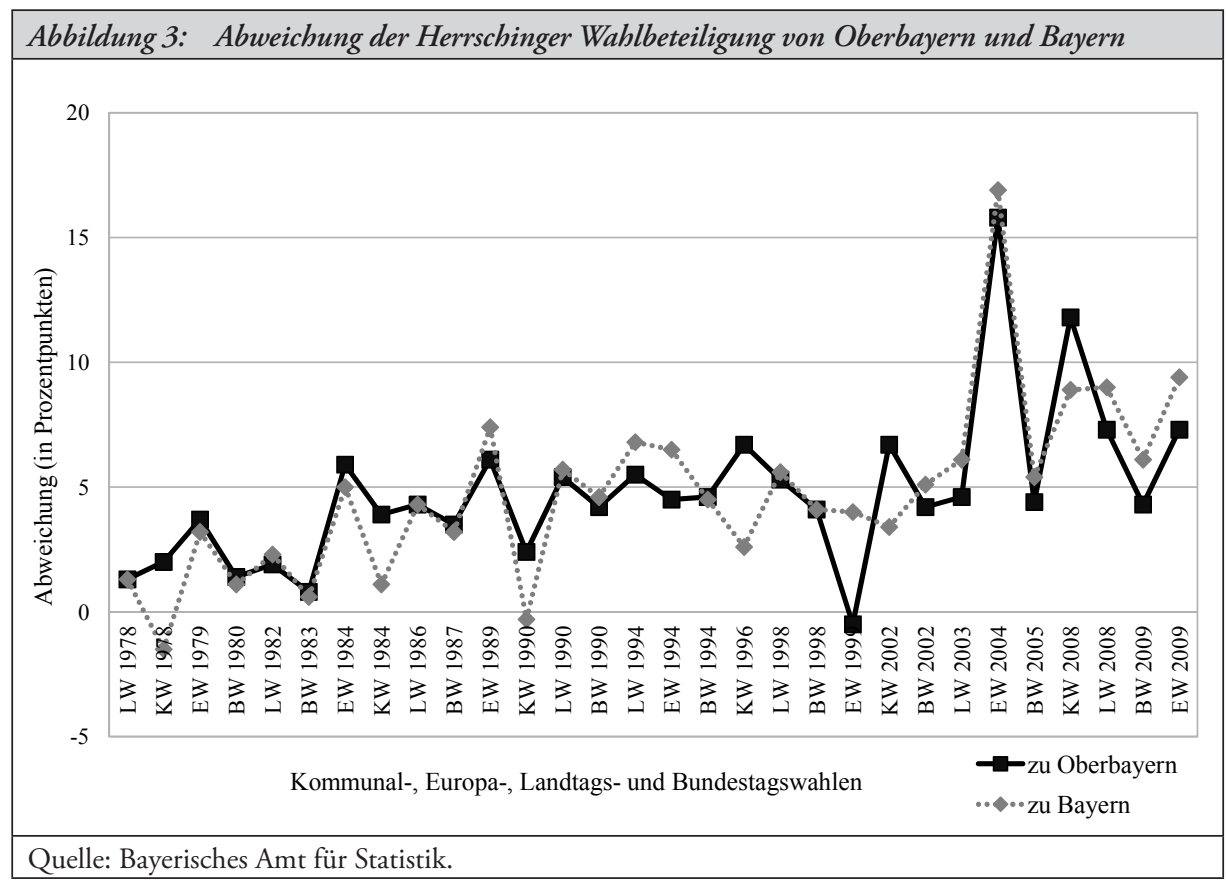


scheiden ihre Stimme abzugeben. Es kann vermutet werden, dass sich dieser positive Effekt auch in den Beteiligungshöhen der folgenden Wahlen niedergeschlagen hat (siehe Abbildung 3). Nach der Europawahl 2004, bei der die Beteiligung im Vergleich zu den Durchschnittswerten in Oberbayern und Bayern mit über 16 Prozentpunkten abwich, lagen auch die folgenden Wahlbeteiligungen auf einem höheren Niveau.

Auf diese Weise entstand aus dem Zusammenspiel von regulärer Wahl und Bürgerentscheid ein positiver Effekt auf die Beteiligungshöhen der nachfolgenden Bürgerentscheide und Wahlen. Somit wurden unterschiedliche Tendenzen in Prien und Herrsching beobachtet (Prien: kontinuierlicher Anstieg; Herrsching: Anstieg nur nach Koppelung mit regulärer Wahl).

\subsection{Politisches Interesse und Engagementbereitschaft}

In beiden Kommunen konnten übereinstimmend keine positiven Auswirkungen auf das Interesse und die Engagementbereitschaft in der Bevölkerung festgestellt werden. So liegt in Prien und Herrsching die Anzahl von Interessierten bei Gemeinderatssitzungen wie vor der Anwendung der Bürgerentscheide bei ein bis fünf Personen. Bei Themen, die für viele von besonderem Belang sind, wie die der Bürgerbegehren, kommen in Prien circa 20 bis 30, in Herrsching circa 40 bis 50 Personen. Diese verlassen die Sitzung jedoch nach dem für sie relevanten Thema wieder, Interesse für andere Themen der Tagesordnung ist nicht vorhanden und scheint auch nicht geweckt zu werden. Die Beteiligung an Gemeindeversammlungen hält sich in Prien seit Jahren konstant bei circa 300 bis 400 Personen, in Herrsching konstant bei circa 150 bis 300, wobei beachtet werden muss, dass für die Ortsteile Widdersberg (circa 100 Personen) und Breitbrunn (circa 50 bis 60 Personen) gesonderte Versammlungen existieren.

Interessant ist die mehrfach geäußerte Beobachtung der Interviewten in Prien, dass dem aktuellen Bürgermeister S. derzeit in diversen Leserbriefen mangelnde Transparenz vorgeworfen wird. Es sei denkbar, dass die Verfasser dieser Leserbriefe ein Bürgerbegehren anstrengten, obwohl gerade dieser Personenkreis zuvor immer gegen die Einleitung von Bürgerbegehren gewesen sei. In diesem Verhalten sind durchaus „Lerneffekte“ zu erkennen, denn diese Personen benutzen nun Partizipationsinstrumente, die ehemals anderen zum Erfolg verhalfen, und derer sie sich selbst in der Vergangenheit nicht bedient hatten. ${ }^{32} \mathrm{Au}-$ ßerdem ist auffällig, dass sich die aktiven Personen der Bürgerbegehren von 1999 und 2005 durchgängig nicht an den Bürgerentscheid von 1997 und das gescheiterte Bürgerbegehren von 1997 erinnern konnten. Dies ist ein Anzeichen dafür, dass das Interesse an kommunaler Politik selbst bei kommunalpolitisch interessierten Personen themenspezifisch aktiviert und ausgelebt wird.

In Prien konnte die Bürgerinitiative „Rettet den Chiemsee“ trotz des starken Engagements einiger ihrer Mitglieder bei den Bürgerbegehren keine Mitgliederzuwächse verzeichnen. Auch in Herrsching, wo sich Gruppen wie die Herrschinger Initiative Trinkwasser (HIT), der Bund Naturschutz und der Agenda 21 Arbeitskreis stark an der Organisation der

32 Tatsächlich wurde 2011 ein Bürgerentscheid durchgeführt, dieser wurde jedoch nicht durch ein Bürgerbegehren initiiert, sondern vom Gemeinderat durch ein Ratsbegehren eingeleitet. 
Bürgerbegehren beteiligt hatten, konnten diese durch ihr Engagement keine weiteren Mitglieder oder Interessierten hinzugewinnen.

In beiden Kommunen waren keinerlei Anzeichen zu erkennen, dass sich durch die Ausweitung des Partizipationsangebots die „Mitwirkungsbereitschaft aller Gemeindeglieder erhöht “33 oder Einwohner für das Gemeinwesen mobilisiert werden konnten. ${ }^{34}$ Beide Kommunen bestätigten damit Andreas Pausts Untersuchungsergebnis, dass durch direktdemokratische Verfahren „die Bürgerschaft zwar mobilisiert, aber nicht aktiviert werden kann“35. Möglicherweise kann eine Aktivierung nur ausgelöst werden, wenn ein entsprechendes Bedürfnis nach mehr Partizipation in der Bevölkerung bereits vorhanden ist. ${ }^{36}$ Auch die Ergebnisse der empirischen Untersuchung von Beilharz, der eindeutig positive Veränderungen im Hinblick auf Interessierte in Gemeinderatssitzungen, Bürgerversammlungen u.ä. feststellt $^{37}$, konnten nicht bestätigt werden.

Dafür gibt es zwei denkbare Erklärungen: Einerseits ist es möglich, dass die Studie von Beilharz durch die sehr viel größere Anzahl von untersuchten Gemeinden genauer ist und die hier ausgewählten Kommunen Einzelfälle darstellen. Andererseits könnten die verschiedenen Ergebnisse durch die unterschiedliche methodische Herangehensweise zustande kommen. Beilharz verwendete standardisierte Fragebögen, die er von den Initiatoren der Bürgerbegehren und den Gemeindeverwaltungen beantworten ließ. Wichen die Antworten in einer Gemeinde stark voneinander ab, führte er persönliche Gespräche. Gab es daraufhin noch immer Diskrepanzen bei den Antworten, wurden diese Gemeinden nicht in die Auswertung einbezogen. Diese Methodik scheint zunächst wenig fehleranfällig zu sein. Es kann jedoch mit den Erfahrungen aus den Interviews in Prien und Herrsching angenommen werden, dass der positive Effekt nur temporär im Diskussionszeitraum des Bürgerentscheids eintrat und nicht, wie später aus Beilharz Ergebnissen geschlossen ${ }^{38}$, von längerer Dauer war. So soll es beispielsweise eine deutliche Erhöhung der Zuhörer in Gemeinderatssitzungen, der Bürger bei Bürgerversammlungen oder der Leserbriefe zu kommunalpolitischen Inhalten gegeben haben. ${ }^{39}$ Diese Veränderungen werden während der Begehren und der Entscheide aufgetreten sein; solche Effekte wurden auch durch die Untersuchung in Prien und Herrsching bestätigt. Hier zeigte sich jedoch, dass diese Effekte zeitlich auf das jeweilige Begehren beschränkt blieben: Das heißt, dass Personen nur in den Gemeinderat gingen, um sich über die Thematik des Bürgerbegehrens zu informieren; dass Bürgerversammlungen deshalb gut besucht waren, weil es um das Thema des Bürgerentscheids ging und dass Personen Leserbriefe schrieben, um ihre Meinung zum Bürgerbegehren kundzutun. Dieser Effekt war aber vorbei, sobald der Bürgerentscheid abgehalten worden war. Berücksichtigt man weiterhin, dass Beilharz 1981 per Fragebogen die Auswirkungen von Begehren erhob, die zum Teil

33 Günter Beilharz, a.a.O. (Fn. 2), S. 137.

34 Vgl. Wilfried Erbguth, a.a.O. (Fn. 1), S. 134; Hans-Herbert von Arnim, a.a.O. (Fn. 1), S. 50.

35 Andreas Paust, Vom Bürgerbegehren zur Bürgergesellschaft, in: APuZ, B 28/2000, S. 22 - 30, S. 23.

36 Vgl. Bettina Knaup, Plebiszitäre Verfahren als Ergänzung der repräsentativen Demokratie. Zur neueren Forschungsdebatte um Volksabstimmungen in der Bundesrepublik Deutschland, Bonn 1994, S. $39-44$.

37 Vgl. Günter Beilharz, a.a.O. (Fn. 2), S. $135 \mathrm{ff}$.

38 Vgl. Stefanie Lackner, a.a.O. (Fn. 11), S. 97 f; Andreas Paust, Direkte Demokratie in der Kommune. Zur Empirie von Bürgerbegehren, Bonn 1999, S. 160.

39 Vgl. Günter Beilharz a.a.O. (Fn. 2), S. 135 f. 
schon 25 Jahre zurücklagen, so sind fehlerhafte Antworten durchaus denkbar. Dies gilt sowohl für die Gemeindeverwaltung als auch für die Initiatoren, weswegen diese Antworten mit Vorbehalt zu betrachten sind.

\section{Bürgerbegehren und -entscheide: Alle Macht dem Volk?}

Da es bei Bürgerbegehren und -entscheiden darum geht, die repräsentative Demokratie durch plebiszitäre Instrumente zu verbessern, ist deren Leistungsfähigkeit von großer Bedeutung. Tatsächlich konnten in den untersuchten Fällen Phänomene beobachtet werden, die stark bezweifeln lassen, ob Bürger durch die direktdemokratischen Instrumente tatsächlich immer in die Lage versetzt werden, den jeweiligen Sachverhalt in ihrem Sinne zu entscheiden.

In Herrsching waren die Bürger- und Ratsbegehren in den Jahren 1997, 2001 und 2004 vom Kalkül der Initiatoren geprägt. Alle Begehren behandelten im Grunde dasselbe Thema: Stellt man einen sensiblen Landschaftsbereich weiterhin unter Naturschutz oder lockert man diesen Schutz zugunsten von wirtschaftlichen Interessen. Über diese Frage wurde jedoch nie direkt abgestimmt, sondern sie wurde immer mit anderen Fragen „kaschiert“ - wahrscheinlich um sich durch dadurch möglichst viele Stimmen zu sichern. Anstatt klar in der Gemeinde die Frage nach dem Willen für ein Naturschutzgebiet und/oder nach der Ausweitung des Gewerbegebiets mit allen Vor- und Nachteilen zu stellen, wurde stattdessen die Frage nach Eigenwasserversorgung oder notwendiger Gewerbeförderung gestellt. Möglicherweise ist dies auch der Grund dafür, dass der gleiche Gegenstand dreimal in einen Bürgerentscheid münden konnte. Es stellt sich hier vor allem die Frage, ob die Einführung direktdemokratischer Instrumente nicht gleichzeitig die Bereitschaft zur umfassenden Offenlegung aller wichtigen Aspekte des Streitthemas verlangt, damit die Bevölkerung sämtliche Pro- und Contra-Argumente abwägen kann und später nicht Verdruss über die unzureichende Informationspolitik herrscht. Dies setzt aber voraus, dass die Bevölkerung als mündiger Souverän verstanden wird, der fähig ist, eine selbstbestimmte Entscheidung zu treffen.

Vor dem Hintergrund taktischen Handelns muss auch die Thematik der Verknüpfung unterschiedlicher Fragen im Antragstext erwähnt werden, die in Prien zu einer absurden Situation führte. Der Begehrenstext der Initiativgruppe lautete: „Soll der gesamte Wendelsteinplatz Grünzone werden, mit einer öffentlichen Tiefgarage, und von einer oberirdischen Bebauung frei bleiben?" Sollte ein Bürger der Meinung sein, dass der Platz eine Grünzone bleiben und auch keine Tiefgarage gebaut werden sollte, dann hätte er mit der Formulierung der Frage im Antragstext ein Problem: Stimmte er dafür, so stimmte er auch für die Tiefgarage, stimmte er dagegen, so stimmte er zugleich gegen den Erhalt der Grünzone. Dieses Dilemma wurde zusätzlich dadurch auf die Spitze getrieben, dass a) nach dem Entscheid ein privater Investor bereit gewesen wäre, die Parktiefgarage unter der Bedingung des Wegfalls aller kostenlosen Parkplätze in Prien zu bauen (was wahrscheinlich nicht unbedingt im Sinne der Bürger gewesen wäre) und b) wahrscheinlich alle hohen Bäume des Wendelsteinplatzes beim Bau der Tiefgarage hätten gefällt und neu angepflanzt werden müssen (was wahrscheinlich ebenso wenig im Sinne der Befürworter der Grünzone gewesen wäre). Faktisch wurde der Bürgerentscheid aus Geldmangel nie umgesetzt, was im Nachhinein wahrscheinlich die beste Lösung, auch für die damaligen Befürworter von Parkhaus und Grünanlage, darstellt. 
Auch in Herrsching zeigte sich eine ungünstige Begleiterscheinung. Beim Bürgerentscheid im Jahr 2004 trat der Fall ein, dass die bindende Entscheidung über den Standort des Gewerbegebiets die Grundstückspreise dort verteuerte, da die Gemeinde die Grundstücke nun in jedem Fall kaufen musste. Ohne Bürgerentscheid hätte über den Preis zunächst intern verhandelt werden können.

Vor diesen Problemen erscheint das Argument vieler Befürworter von Bürgerentscheiden, durch sie würden die Bürger in die Lage versetzt werden, selbst über ihre Interessen abstimmen zu können, in einem ganz anderen Licht: Tatsächlich kann die Bevölkerung bei Bürgerentscheiden nur insoweit über einen Sachverhalt abstimmen, wie es die Fragestellung des Bürgerentscheids zulässt und wie dies von den Initiatoren des Bürgerbegehrens gewollt ist. Dieses Problem, das in gewisser Hinsicht dem Grundgedanken von direkter Demokratie widerspricht, könnte durch Veränderungen zumindest gelindert werden: Eine Möglichkeit wäre, die Verknüpfung von zwei Fragen zu sanktionieren (was jedoch durch das restriktive Moment nicht unbedingt zum direktdemokratischen Instrument passen würde), eine andere, jede Frage eines Entscheids getrennt voneinander abzustimmen. Nur diejenigen Fragen würden dann positiv beschieden, die von der Mehrheit eine Zustimmung erhalten haben. Ansonsten wird/werden diese aus dem (erfolgreichen) Begehren herausgelöst. Diese Lösungsansätze könnten verhindern, dass „Gesamtpakete“ in den Fragestellungen die Bürger in ihrer eigentlich beabsichtigten Abstimmungsfreiheit beschneiden.

Solche „Gesamtpakete“ können ebenfalls Probleme bei Bürgerentscheiden zu Bauprojekten auslösen. Durch einen Bürgerentscheid werden bauliche Vorgaben gemacht, die später durch den Gemeinderat oder die Verwaltung nicht mehr verhandelbar sind. Sie sind damit zwar Willensausdruck der Bevölkerung, doch ist es später für den Gemeinderat nicht mehr möglich, ausgleichende Kompromisse durch Verhandlungen zu bewirken - beispielsweise, wenn es um Wünsche betroffener Anwohner geht, die durch kleine Änderungen umgesetzt werden könnten und wahrscheinlich auch im Sinne einer guten Atmosphäre für die Gesamteinwohnerschaft wären.

Hier wird besonders die Unfähigkeit direktdemokratischer Instrumente zu konsensualen Entscheidungen deutlich: Bürgerentscheide können zwar Streitthemen durch eine Mehrheitsentscheidung befrieden, die Möglichkeit des Interessenausgleichs ist nach ihrer Einleitung aber nicht mehr gegeben. Aus diesem Grund wäre es ratsam, die Anzahl der benötigten Unterschriften für Bürgerbegehren in Bayern nicht weiter abzusenken (um zu gewährleisten, dass eine relevante Bevölkerungsanzahl hinter den Fragen steht) und mit Bürgerentscheiden nur grundsätzliche Entscheidungen herbeizuführen (für oder gegen ein Bauprojekt) und Einzelheiten der Verwaltung oder dem Gemeinderat zu überlassen. Gerade Letzteres wird jedoch nicht im Interesse von Initiatoren liegen, da das Vertrauensverhältnis zu Verwaltung und Rat zu diesem Zeitpunkt meist bereits so beschädigt ist, dass die Initiatoren bevorzugt jede Einzelheit abstimmen möchten, um hinterher keinerlei Interpretationsspielraum offenzulassen (in Herrsching gipfelte dies bei einem Bürgerbegehren in einen zweiseitigen Fragetext). Die Beispiele zeigen, dass eine frühe und umfassende Einbeziehung der Bürger in kommunale Entscheidungen viel wichtiger und sinnvoller ist, als über Projekte in weitestgehend ausgereiftem Zustand in einem Bürgerentscheid abzustimmen. Direktdemokratische Instrumente sollten aus diesem Grund erst die letzte Möglichkeit der Entscheidungsfindung in einer Kommune darstellen. 


\section{Fazit: Nur temporäre Partizipationserhöhung, aber Möglichkeit zur Steigerung der Wablbeteiligung}

Der Vergleich der Ergebnisse aus den Untersuchungen der Gemeinden Prien am Chiemsee und Herrsching am Ammersee zeigt, dass nicht pauschal von einer dauerhaften Aktivierung politischer Partizipation durch direktdemokratische Verfahren auf der kommunalen Ebene ausgegangen werden kann. Die Angebotserhöhung von Partizipationsmöglichkeiten führt nur zu einer temporären Nachfrageerhöhung. Es gab in beiden untersuchten Gemeinden keinerlei Anhaltspunkte, dass Einwohner zu politischem Engagement oder vermehrtem politischen Interesse über die Bürgerentscheide hinaus aktiviert werden konnten. Nur in Einzelfällen konnte dies bei Initiatoren, die zuvor nicht politisch aktiv gewesen waren, nachgewiesen werden. Außer einem leicht positiven Einfluss auf die Wahlbeteiligung, der sich in einer Kommune vermuten lässt, konnte kein pauschaler positiver Einfluss auf die Wahl- und Abstimmungsbeteiligung festgestellt werden. Allerdings zeigte sich in einer Gemeinde, dass ein Bürgerentscheid, der terminlich mit einer regulären Wahl zusammenfiel, mit dieser gemeinsam eine deutlich positive Auswirkung auf die nachfolgenden Beteiligungen bei Wahlen und Abstimmungen besaß. Die direktdemokratischen Instrumente konnten zwar Veränderungen wie zum Beispiel den Wechsel des Bürgermeisters, die Verbesserung der Arbeitsatmosphäre im Gemeinderat oder die Gründung einer neuen Wählergemeinschaft bewirken; eine Aktivierung politischer Partizipation über die Zeit der Bürgerentscheide hinaus konnte aber nur in sehr geringem Maße festgestellt werden. In den untersuchten Gemeinden ließen sich die größten Veränderungen bei der personellen Besetzung und der Parteizugehörigkeit der Bürgermeister beobachten.

Die Annahme, dass eine größere Anzahl von Partizipationsmöglichkeiten gleichzeitig eine erhöhte Beteiligung bedeutet, kann also nicht aufrecht erhalten werden. Autoren, die aus dieser Annahme heraus direktdemokratischen Instrumenten pauschal erhöhte partizipatorische Wirkungen unterstellen, müssen sich folglich revidieren.

Trotz des Ergebnisses, dass direktdemokratische Verfahren kein Mittel gegen eine niedrige Wahlbeteiligung darstellen, zeigte der Bürgerentscheid 2004 in Herrsching, dass die Kombination aus Bürgerentscheid und regulärer Wahl dazu geeignet ist, die Wahl- und Abstimmungsbeteiligung an diesem Termin und auch bei darauffolgenden Wahlen und Abstimmungen sichtlich zu erhöhen. Es wäre lohnenswert zu überprüfen, ob sich dieses Phänomen auch in anderen Kommunen bestätigen lässt. Hier deutet sich die Möglichkeit an, Wahlbeteiligungen auf kommunaler Ebene gezielt zu erhöhen: Durch den Beschluss von Ratsbegehren könnten die vorhandenen Wahlen mit Bürgerentscheiden über wichtige Themen ergänzt und auf diese Weise die Bürger motiviert werden, sich an Wahlen zu beteiligen. ${ }^{40}$ Eine solche Handhabung würde jedoch eine grundlegende Veränderung der parlamentarisch-repräsentativen Demokratie in Deutschland darstellen. Dennoch stellt sich die Frage, ob ein solcher Zustand nicht viel eher dem Idealbild einer umfassenden Demokratie entspräche, das Adolf Arndt 1958 als „Selbstbestimmung mündiger Menschen und die bestmögliche Verwirklichung dieser Selbstbestimmung " 41 beschrieben hat.

40 Vgl. dazu kritisch Werner J. Patzelt, a.a.O. (Fn. 7), S. 97 - 101.

41 Adolf Arndt zitiert nach Rudolf Wassermann, Plebiszitäre Demokratie - ja oder nein?, in: Recht und Politik, B 22/1986, S. 125 - 131, S. 126. 